\title{
Enunciación
}

http://revistas.udistrital.edu.co/ojs/index.php/enunc

DOI: http://dx.doi.org/10.14483/udistrital.jour.enunc.2016.2.a02

enunciación

Artículo de REFLEXIÓN

\section{L'œuvre littéraire et le réel: entre l'ambition de saisir le monde et le défi de la création}

\author{
La obra literaria y la realidad: entre la ambición de apoderarse del mundo y el \\ desafío de la creación
}

\section{The literary work and the real: Between the ambition of grasping the world and the challenge of creation}

\author{
Diana Marcela Patiño Rojas ${ }^{1}$
}

Para citar este artículo: Patiño Rojas, D.M. (2016). L’œuvre littéraire et le réel: entre l’ambition de saisir le monde et le défi de la création. Enunciación, 21(2) 201-211.

Recibido: 29-abril-2016 / Aprobado: 24-agosto-2016

\section{Résumé}

Cet article propose une réflexion à propos de la nature du lien entre l'oeuvre littéraire et le réel, à travers l'analyse de différentes perspectives théoriques présentées par des philosophes, écrivains et théoriciens de la littérature tels que Platon, Aristote, Diderot, Hugo, Balzac, Champfleury, Maupassant, Baudelaire, Cousin, Proust et Barthes., appartenant à différentes époques. II s'agit donc de retracer les conceptions de ces auteurs par rapport à l'oeuvre littéraire et son lien avec le réel avec le double propos de definir un panorama de la question, dans lequel l'on met en évidence le développement d'une discussion qui a eu lieu pendant des siècles; et de présenter un point de vue particulier afin de nourrir cette réflexion. Egalement, l'on cherche à constater que les considérations sur le réel et son rapport avec l'oeuvre, continuent d'être de nos jours, l'objet de réflexion et de la création littéraire.

Mots clés: réel, réalité, œuvre littéraire, écriture.

\begin{abstract}
Resumen
En el presente artículo se propone una reflexión sobre la naturaleza de la relación entre la obra literaria y lo real, a través del análisis de diferentes posturas teóricas de filósofos, escritores y estudiosos de la literatura de diferentes épocas como Platón, Aristóteles, Diderot, Hugo, Balzac, Champfleury, Maupassant, Baudelaire, Cousin, Proust y Barthes. Desde esta perspectiva, se trata de revisar de manera crítica las concepciones de estos autores con respecto a la obra literaria y su vínculo con la realidad, con el doble propósito de definir un panorama de la cuestión en el que se evidencie el desarrollo de una discusión que ha tenido lugar durante siglos y de presentar un punto de vista propio que alimente la reflexión. Así mismo, se busca demostrar que las consideraciones sobre la realidad y su relación con la obra continúan siendo hoy en día objeto de reflexión y creación literaria.
\end{abstract}

Palabras clave: realidad, obra literaria, escritura.

1 Profesora de la Escuela de Ciencias del Lenguaje de la Universidad del Valle. Correo electrónico: diana.marcela.patino@correounivalle.edu.co 


\begin{abstract}
The present article proposes a reflection about the nature (character) of the relationship between the literary work and reality taking into account different theoretical stances from philosophers, writers and literature scholars such as Plato, Aristotle, Diderot, Hugo, Balzac, Champfleury, Maupassant, Baudelaire, Cousin, Proust and Barthes. According to this perspective, the idea is to look over these author's conceptions about literary work and their connection with reality in order to
\end{abstract}

\section{INTRODUCTION}

Le rapport entre l'œuvre littéraire et le réel a toujours été l'objet d'une réflexion qui a marqué, à travers les époques, le développement des manifestations artistiques dans le domaine des lettres en Occident. De la tradition grecque à nos jours en passant par le Moyen Age et la modernité, l'esthétique du réel se transforme et se renouvelle afin de rendre compte des drames de l'être humain dans une société qui semble l'anéantir. Dans ce sens, il s'avère important dans le cadre des études littéraires, de s'interroger sur la nature du lien entre l'œuvre et le réel et d'explorer les différentes postures théoriques qui existent à ce propos.

Cet article tentera donc d'analyser cette question en s'appuyant sur l'analyse de diverses conceptions de remarquables écrivains et théoriciens comme Platon, Aristote, Diderot, Hugo, Balzac, Champfleury, Maupassant, Baudelaire, Cousin, Proust et Barthes, afin de retracer les réflexions les plus importantes qui ont nourri la discussion en enrichissant l'ensemble de références sur le rapport entre l'œuvre et le réel.

L'on verra par exemple que pour les philosophes grecs, l'imitation du réel se trouve au cœur de la fiction littéraire même s'ils ne lui accordent pas la même valeur. Puis, au moyen Age, en revanche, la question a été mise de côté mais elle a été reprise par des théoriciens comme Diderot lors du siècle des Lumières. define an outlook in which it is possible to make clear the development of this dialogue carried out for centuries. The aim of this study is also to introduce another point of view about the question for encouraging the discussion. Likewise, the final phase is to validate the considerations about reality and his connection with literary work are still the object of many reflections and the source of literary creation.

Keywords: reality, literary work, writing.

Au XIX siècle, le débat sur l'œuvre et le réel conduit à spécifier la relation existante entre ces deux notions tout en proposant une esthétique particulière qui correspond au Réalisme en tant que mouvement et enfin au XX siècle, ces formes littéraires reviennent renouvelées pour témoigner de l'ambition récurrente de la littérature de s'emparer du réel, de le reproduire et pourquoi pas le modifier.

Pour conclure, I'on montrera dans le présent travail que la réflexion sur le rapport entre l'œuvre littéraire et le réel ne cesse pas d'être alimentée encore de nos jours.

\section{L'OEUVRE LITTÉRAIRE ET LE RÉEL: À L'ORIGINE DE LA QUESTION}

La réflexion sur l'œuvre littéraire et son rapport avec le réel a été à l'origine d'un débat mené depuis la Grèce Antique. La question a été posée par Platon, philosophe qui dans une perspective idéaliste fait référence, surtout dans le livre $X$ de la République, à la notion d’imitation liée à l'art de l'écriture. D’après lui, le fait d'imiter fournirait une image dégradée de ce qui n'est qu'une impression de l'artiste, c'est-à-dire, le reflet d'une idée. Pour Platon donc, la démarche littéraire qui cherche à imiter la nature humaine n'atteint pas la vérité mais ne saisit que des apparences. D’où la sévère condamnation des poètes, non seulement dans la République mais dans Phèdre et le Sophiste, à 
partir de ce principe fondamental : "n'admettre, en aucun cas, ce qui dans la création poétique est imitation" (Platon, 1991, p. 625).

Cependant Aristote, à l'opposé de son maitre Platon, met en valeur l'imitation (mimesis) et suggère, dans la Poétique, que ce procédé est la base des expressions littéraires de l’époque. Pour cet auteur classique, la tragédie est donc :

L'imitation d'une action noble, conduite jusqu'à sa fin et ayant une certaine étendue (...); C'est une imitation faite par des personnages en action et non par le moyen d'une narration, et qui par l'entremise de la pitié et de la crainte, accomplit la purgation des émotions de ce genre (Aristote, 1990, pp. 92-93).

Ainsi, l'imitation est revendiquée par Aristote comme la source d'inspiration de la création littéraire dont le résultat est une œuvre censée opérer une purgation (catharsis) des émotions chez le spectateur et susciter une réflexion sur la nature humaine. Dans cette perspective, la représentation théâtrale axée sur le principe de l'imitation, permettrait de récréer des sentiments liés aux faiblesses intérieures afin de mieux les gérer dans la vie réelle.

Cette conception aristotélicienne de la mimesis prend alors de l'ampleur et se situe au cœur de la création littéraire et artistique de l'époque en distinguant deux types d’imitation : la simple imitation de la nature et la stylisation de celle-ci. Cela veut dire, que l'écrivain a pour but de dépasser la description et forger une esthétique particulière capable de rendre compte des meurs de la société. D’où l'importance de l'observation et la réflexion sur l'entourage en tant que moteur créateur.

Néanmoins, au Moyen Age, la question du réel dans l'œuvre littéraire est écartée et d'autres formes esthétiques voient le jour. Pendant cette période, la plupart des ouvrages représentaient des motifs théologiques et la littérature d'inspiration religieuse est donc la plus répandue. Toutefois, d'autres genres, assez liés aux expériences de la vie quotidienne se développent tels que les chansons de geste, le roman courtois, les farces, les satires et le roman d'aventures, entre autres. Ce qui montre que l’intérêt de recréer le monde à travers les manifestations littéraires n’a pas disparu.

Or, ce n'est que pendant la période de la Renaissance que le rapport entre l'œuvre et le réel retrouve peu à peu son essor, grâce aux nouvelles approches culturelles issues des modèles grecs et latins qui se consolideraient plus tard, pendant le siècle des lumières. A cette époque-là, Diderot reprend la réflexion sur le réel de façon concrète dans l'Eloge de Richardson, critique littéraire qui porte sur Samuel Richardson, auteur de Clarisse Harlowe. Dans ce texte, Diderot conçoit "I'unité profonde, dans son approche du romanesque, entre l'exigence de vérité, la pédagogie morale et la réussite esthétique" (Duflo, 2013, p. 8) affirmant que :

Cet auteur [Richardson] ne fait pas couler le sang le long des lambris; il ne vous transporte point dans des contrées éloignées; il ne vous expose point à être dévoré par des sauvages; il ne se perd jamais dans les régions de la féerie. Le monde ou nous vivons est le lieu de la scène; le fond de son drame est vrai ; ses personnages ont toute la réalité possible ; ses caractéristiques sont prises du milieu de la société ; ses incidents sont dans les mœurs de toutes les nations policées ; les passions qu'il peint sont telles que je les éprouve en moi (Diderot, 1761, p. 6).

Ainsi, Diderot valorise le décor réaliste sur le plan de I'histoire dans le texte littéraire et associe la notion du réel à celle de vérité. L'on a l'impression que pour cet auteur, ce qui est "réel" est "vrai" et vice-versa, idée qui élargie la question et l'ouvre également à la problématique théorique de la vérité afin d'enrichir le débat.

De la même manière, Diderot exalte le fait que les personnages et leurs caractéristiques correspondent aux référents de la société et incarnent les mœurs et les passions proches de l'individu de l'époque. De ce fait, l'on constate que l'auteur propose un vrai éloge du réel en tant qu'élément fondamental de l'esthétique littéraire qui permet 
de développer une littérature très proche du vécu humain à travers I' observation et l'exploration d’un milieu social susceptible d’être appréhendé.

\section{LE RÉEL À LA BASE D’UN MOUVEMENT LITTÉRAIRE}

Ensuite, au XIX siècle, bien que l'esthétique classique et même romantique considéraient que "I’imitation de la nature et du milieu social n'est pas art, car l'art doit viser au beau idéal" (Mizuno, 2005, p. 822), l'écriture du réel se consolide dans un courant propre. Les phénomènes historiques et sociaux deviendront le support de l'œuvre littéraire grâce à la profonde volonté des auteurs de faire coïncider la fiction avec divers aspects de la vie sociale et quotidienne. Ainsi, I'on constate qu'un bon nombre de textes ont été conçus à partir d'un regard "réaliste", ce qui conduit à préciser d'un point de vue théorique et esthétique, la relation qui existe entre l'œuvre littéraire et le réel.

A cet égard Victor Hugo proclame dans la Préface de Cromwell, d'ailleurs bien avant les précurseurs du réalisme, que "tout ce qui est dans l'art est dans la nature" (Hugo, 1827, p. 301), constat qui suggère l'œuvre comme un espace infini ou la nature prend forme ainsi que dans le monde réel. Cet écrivain prend l'exemple du drame et ajoute que celui-ci est un miroir où se réfléchit la nature, métaphore que reprendra plus tard Stendhal ${ }^{2}$ et Balzac, détenteur lui aussi de propos similaires envers l'œuvre et le réel. Dans cette perspective, Hugo précise :

Si ce miroir est un miroir ordinaire, une surface plane et unie, il ne renverra des objets qu'une image terne et sans reliefs, fidèle, mais décolorée ; on sait ce que la couleur et la lumière perdent à la réflexion simple. Il faut donc que le drame soit un miroir de concentration qui, loin de les affaiblir, ramasse et condense les rayons colorants, qui fasse d'une lueur une lumière, d'une lumière, une flamme...tout ce

2 Un roman est un miroir qui se promène sur une grande route. Tantôt il reflète à vos yeux l'azur des cieux, tantôt la fange des bourbiers de la route (Stendhal, 1972, p. 19). qui existe dans le monde, dans l'histoire, dans la vie, dans I'homme, tout doit et peut s'y réfléchir, mais sous la baguette magique de l'art (Hugo, 1827, p. 302).

Ce positionnement vis-à-vis de la littérature et son rapport avec le réel consolide l'idée d'une réalité à mettre "en lumière" à travers une démarche esthétique spécifique tout en exprimant un double propos : d'une part, il s'agit d'une invitation à la réflexion profonde sur l'humain qui passe avant tout pour le besoin de contempler et analyser notre entourage entendu comme la réalité qui nous concerne et nous accable ; et d'autre part, que l'art a un caractère universel permettant $d^{\prime}$ explorer tous les aspects de l'existence.

Par ailleurs, mais toujours dans la même perspective Balzac exprime :

L'art littéraire, ayant pour objet de reproduire la nature par la pensée, est le plus compliqué de tous les arts. (...) L'écrivain doit être familiarisé avec tous les effets, toutes les natures. Il est obligé d'avoir en lui je ne sais quel miroir concentrique où, suivant sa fantaisie, l'univers vient se réfléchir ; sinon, le poète et même l'observateur n'existent pas ; car il ne s'agit pas seulement de voir, il faut encore se souvenir et empreindre ces impressions dans un certain choix de mots et les parer de toute la grâce des images ou leur communiquer le vif des sensations primordiales (Balzac, 1831, p. 309).

Cette métaphore du miroir constitue donc le point de départ d'une réflexion plus élargie basée sur le rôle de l'écrivain, en tant qu'observateur et artiste, son rapport avec le monde et la valeur esthétique de la langue.

Dans ce sens, Balzac met en évidence que le questionnement sur l'œuvre littéraire et le réel implique, d'ailleurs, une prise en compte des instances narratives et théoriques devenant ainsi un des précurseurs du mouvement réaliste du XIX siècle. Ce courant littéraire s'inscrivant tout au début de la Deuxième République (24 février 1848) jusqu'à la fin du Second Empire (septembre 1870), a pour but de "représenter objectivement la vie quotidienne ce qui correspond à un besoin 
de vérité en s'opposant à une autre tendance qui souhaite donner une vision flattée, embellie ou déformée de la réalité" (Stalloni, 2011, p. 112). Ainsi, étant le milieu social le thème privilégié du mouvement, Balzac, toujours dans la même démarche littéraire, propose le concept de Comédie Humaine "le tableau exact des mœurs" afin de donner dans ses textes, une impression de vérité en s'appuyant sur la vie réelle.

De la même manière Champfleury, dans un ouvrage intitulé Le Réalisme, définit la théorie du mouvement comme" La reproduction exacte, complète, sincère du milieu où l'on vit, parce qu'une telle direction d'études est justifiée para la raison, les besoins de l'intelligence et l'intérêt public, et qu'elle est exempte de mensonges, de toute tricherie" (Champfleury, 1857, p. 91). Cela insiste sur la préférence de l'écrivain pour le réel et le rejet du romanesque qui évoque une sensibilité dépourvue de toute objectivité. Ainsi, seIon Champfleury, le mouvement conçoit l'œuvre littéraire comme le résultat d'un travail objectif d'observation orienté par la raison qui aboutit à la création d'une image nette de la société au service des gens. Ces préceptes de base s'avèrent fondamentaux dans la mesure où la conception de l'œuvre s'élargit en intégrant une vocation sociale qui ne se trouve pas contemplée dans d'autres esthétiques qui prônent avant tout l'idéal de beauté. Dans cette optique, le réel, en tant qu'élément de la poétique, se consolide également comme un outil dont la société peut se servir pour explorer et capter son propre milieu.

C'est pourquoi, bien évidemment, il ne s'agit pas de décrire. D’après Maupassant (1888) :

Le réaliste, śil est un artiste, cherchera non pas à nous montrer la photographie banale, ou à nous en

3 La Société française allait être I'historien, je ne devais être que le secrétaire. En dressant l'inventaire des vices et des vertus, en ressemblant que les principaux faits des passions, en peignant les caractères, en choisissant les événements principaux de la Société, en composant des types par la réunion des traits de plusieurs caractères homogènes, peut-être pouvais-je arriver à écrire I'histoire oubliée par tant d'historiens, celle des mœurs (Balzac, 1842 , p. 2). donner la vision plus complète, plus saisissante, plus probante que la réalité même. (...) Faire vrai consiste donc à donner l'illusion complète du vrai, suivant la logique ordinaire des faits et non à les transcrire servilement dans le pêle-mêle de leur succession. J'en conclus que les Réalistes de talent devraient s'appeler plutôt des illusionnistes (p. 10).

D’où le paradoxe de l'illusion du réel qui est à l'origine de la démarche créatrice d'une littérature qui cherche quand même à "dépasser la simple description de la société pour en démontrer les mécanismes" (Becker, 2005, p. 51). D’ailleurs, cette idée d'illusion ancrée consciemment dans le réel, met en évidence que la référence du réel dans l'œuvre littéraire est avant tout une fiction. Cette manière de s'emparer du réel révèle que la réalité n'est pas donc seulement un ensemble de faits ou d'expériences mais le résultat d'une élaboration cognitive et esthétique revêtue de l'essence de soi.

Dans ce sens, le mouvement prône une méthode d'écriture dont le principe fondamental est I'observation : les faits et situations seront établis à partir de l'observation exhaustive. Puis la mise en récit entraine non seulement une narration qui rend compte des évènements mais des détails de la scène, du décor qui encadre l'action. Cette sorte de description permettant au lecteur de se figurer de manière très claire le contexte mais qui ne semble avoir aucune fonction dans l'intrigue, constitue ce que Barthes appellerait plus tard l'effet du réel (1968). Dans une époque où les études du récit littéraire se concentraient sur la structure, cet auteur met en valeur les éléments d'ordre descriptif et remarque leur importance dans le sens où ils aboutissent à la vraisemblance et consolident le rapport entre le texte et le réel. D’où leur fonction dans I'œuvre littéraire. D’ailleurs, Barthes cite un extrait du Cour simple de Flaubert, afin de théoriser ce concept et de constater que "I'exactitude dans la description d'un réfèrent est à la base de la démarche esthétique qui évite de se laisser entrainer dans une activité fantasmatique" (Barthes, 1968, p. 87). 
Cependant, ce souci d'exhaustivité dans la description du monde concret présent dans un bon nombre d'œuvres littéraires du XIX siècle, se révèle problématique pour certains écrivains qui expriment également leur point de vue sur la question. Victor Cousin par exemple, dans son article sur l'esthétique du Beau conteste la valeur de l’art attachée au réel en proposant ce questionnement par rapport à l'artiste et son œuvre :

Si se borne-t- il à les reproduire [les éléments du réel] tels que la nature les lui fournit, n'est-il que le copiste de la réalité, son seul mérite est alors celui de la fidélité de la copie. Mais quel travail plus stérile que de calquer des œuvres essentiellement inimitables, pour en tirer un simulacre médiocre? (Cousin, 1845, p. 793).

Cette interrogation relève deux aspects fondamentaux du débat à savoir l’impossibilité de reproduire le réel dans l'œuvre d'art et la notion de copie dans le domaine artistique en lui attribuant une connotation négative.

De la même manière, Baudelaire coïncide avec Cousin sur le fait que l'art ne doit pas se borner à chercher la représentation fidèle du réel. Le poète affirme, dans un compte rendu du salon de 1859, que "de jour en jour l'art diminue le respect de luimême, se prosterne devant la réalité extérieure" (Baudelaire, 1859, p. 619), critiquant ainsi les doctrines artistiques, notamment les lettres et la peinture, qui se donnent pour idéal de rendre compte du réel tel qu'il est. Lui-même en revanche exalte l'imagination et la considère reine des facultés humaines, idée qui marquera un des principes fondamentaux de la poésie moderne en occident développé non seulement par ce poète mais par d'autres écrivains comme Rimbaud et Verlaine, entre autres.

Ensuite, grâce à Marcel Proust, la question du réel dans l'œuvre littéraire acquiert une nouvelle nuance et se pose donc en des termes nouveaux. Pour l'auteur de $A$ la recherche du temps perdu, la perception de la réalité est fondamentalement subjective :
La littérature ne peut pas se contenter de saisir I'apparence des objets car la littérature qui se contente de "décrire les choses ", d'en donner seulement un misérable relevé de lignes et de surfaces, est celle qui, tout en s'appelant réaliste, est la plus éloignée de la réalité, celle qui nous appauvrit et nous attriste le plus, car elle coupe brusquement toute communication de notre moi présent avec le passé, dont les choses gardaient l'essence, et l'avenir, ou elles nous incitent à la goûter de nouveau (Proust, 1927, p. 885).

Dans ce sens, nos impressions du moment sont liées aux objets du monde, et les souvenirs qui nous en restent conservent l'essence de notre moi. La réalité brute, immédiate, que l'on pourrait décrire n'a donc aucune valeur. "L'écrivain ne peut faire l'économie d'un travail d'interprétation et d'écriture" (Vassivière et Toursel, 2011, p. 46).

Ainsi, se démarquant de l'idée du réalisme pure et transparent, Proust coïncide avec Hugo, Balzac et Maupassant sur le fait que la démarche créatrice de la littérature doit dépasser la description détaillée du monde et enrichit le débat avec une redéfinition de la réalité qui intègre le temps et la mémoire et leur influence sur la manière dont on perçoit le réel immédiat. La perception de la réalité se développe donc en fonction des rapports humains avec le temps et avec le sens dont les choses sont revêtues selon nos expériences. De ce fait, l'écrivain est censé rendre compte du réel, ou plutôt de son réel, en faisant une sorte de tableau emmaillé de son essence et de sa perception des choses.

\section{FICTIONS CONTEMPORAINES : LE RÉEL POSTMODERNE}

Or, l'évolution de la modernité n'a pas déplacé la question du lien entre l'œuvre littéraire et le réel, bien au contraire, cette thématique est abordée avec acuité par de nombreux écrivains et chercheurs comme Simone de Beauvoir et Jean-Paul Sartre qui vers la première moitié du XX siècle, construisent tout un ensemble d'œuvres littéraires 
remarquables dont la raison d'être est l'engagement à représenter le monde en illustrant la société qui le compose. Pour ce dernier, la fonction de l'écrivain est de "faire en sorte que nul ne puisse ignorer le monde et que nul ne s'en puisse dire innocent" (Sartre, 1948, p. 30), réflexion qui insiste sur le double propos de la littérature : d'une part, faire connaitre le monde en combattant l'ignorance de ses phénomènes sociaux ; et d'autre part, s'engager en contestant les situations illustrées à travers les œuvres.

La littérature donc, en tant que moyen de diffusion, créatrice de conscience individuelle et collective, attrape une réalité fuyante (Sartre, 1948, p. 20) et un mouvement, tout en nous permettant de nous l'approprier et d'agir sur elle.

Par ailleurs, cette conception de littérature engagée se voit contestée par les idées d’Alain Robbe-Grillet, théoricien et représentant du Nouveau Réalisme, qui atteste que "I'écriture romanesque ne vise pas à informer, comme le fait la chronologie, le témoignage ou la relation scientifique. Elle constitue la réalité" (Robbe-Grillet, 1961, p. 138). Ainsi, il y aurait un réel littéraire autonome et indépendant qui se développerait au sein de l'œuvre de manière systématique et qui autoriserait la littérature à se détacher du réel objectif afin de créer son propre monde possible.

Cette position, suggérant la possibilité d'une écriture romanesque capable de constituer une réalité en elle-même, trouve une place très importante dans le champ littéraire français pendant les années soixante. Toutefois, en 1962 Georges Perec s'y opposerait en précisant que la tâche de la littérature implique une "mise en ordre du monde" qui passe par "l'expression la plus totale des réalités concrètes" à travers la description. Donc, pour cet auteur "décrire la réalité c'est plonger en elle et lui donner forme" (Perec, 1992, p.51).

De cette façon, en opposition avec l'idée de Robbe-Grillet, la théorie de Perec envisage un réel littéraire subordonné aux réalités concrètes de la société, susceptible d'être exploré, compris et expliqué à travers la littérature. Ceci dit, il s’agit de concevoir un exercice d'écriture qui chercher à s'emparer du monde et le reconfigurer.

Puis, dans les années quatre-vingt, une nouvelle génération d'écrivains français (Duras, Kaplan, Bon, Martin, Rolin, Le Dantec, Goux, Gracq et tant d'autres), reprend les éléments stylistiques d'une poétique réaliste consolidant une exploration romanesque renouvelée qui donne naissance aux nouvelles formes littéraires dont le propos est également rendre compte du réel. Ces œuvres, dont la plupart sont des romans, comme au XIX siècle, bien que différentes dans leur style, partagent une formule narrative de base : s'inspirer du milieu social afin de créer une image fidèle de la société et de ses drames.

Un courant réaliste contemporain se développe donc basé, de manière générale, sur des principes comme la description des mutations sociales, la reprise de la problématique du monde du travail, l'énonciation de la violence et les fractures individuelles et collectives (Viart et Vercier, 2008, p. 220).

Dans ce domaine, des auteurs remarquables comme François Bon, Nathalie Kuperman, Leslie Kaplan, Gérard Mordillat et Delphine de Vigan ont fait du monde contemporain du travail leur objet de réflexion et d'écriture. Ainsi à travers des projets énonciatifs différents, ces écrivains cherchent à consolider une esthétique réaliste totalement renouvelée caractérisée par l'intégration des références historiques et journalistiques au sein du texte (ce qui permet de créer un réseau textuel à l'intérieur de l'ouvre), la description détaillée des espaces géographiques et des atmosphères du travail, et le caractère discontinu des récits :

L'usine, la grande usine univers, celle qui respire pour vous. Il n'y n'a pas d'autre air que ce qu'elle pompe, rejette. On est dedans. Tout l'espace est occupé : tout est devenu déchet. La peau, les dents, le regard. On circule entre des parois informes. On croise des gens, des sandwichs, des bouteilles de co$\mathrm{ca}$, des instruments, du papier, des caisses, des vis. On bouge indéfiniment, sans temps. Ni début, ni fin. 
Les choses existent ensemble, simultanées. A l'intérieur de l'usine, on fait sans arrêt. On est dedans, dans la grande usine univers, celle qui respire pour vous (Kaplan, 1982, p. 10).

La réalité de l'usine : lieu impersonnel et de non-sens où l'on vit et l'on meurt. Et pourtant, le "je" ne retrouve pas sa place. Bien au contraire, le sujet se dessine à travers un discours fragmentaire et disloqué qui montre à quel point la société française post-industrielle est en crise. Une crise qui se nourrit, d'une part de l' absence de sens dans le monde du travail ; et d'autre part du grand nombre de licenciements qui ont lieu tous les jours en France. En effet, ce phénomène social est l'objet de récit de nombreux romans dont Les Vivants et Les Morts de Mordillat et Nous Nous Etions des êtres vivants de Kupperman, histoires qui mettent en évidence comment le désespoir de se retrouver sans emploi pousse les gens à la violence :

$C^{\prime}$ est très simple, crie-t-il pour se faire entendre. Ceux qui veulent quitter la Kos, quittent la Kos [l'usine]. Mais ceux qui restent doivent savoir que nous ne céderons pas. Nous sommes décidés à aller jusqu'au bout. S'il faut faire péter les machines une par une, nous le ferons péter ! II n'est pas question de les laisser partir [...] le liquidateur peut se brosser (Mordillat, 2004, p. 614).

Quant à l'écrivain, celui-ci assume le rôle d'un ethnographe, qui au-delà de son travail d'observation, met en scène l'impotence et l'inégalité de la lutte face à la mondialisation et à l'économie libérale :

Au départ non pas de personnages, mais seulement des images, que je n'arrive pas à rejoindre. Liées seulement par une hantise, une peur. [...] Le seul passage possible pour moi, au bout d'années sans déboucher, aurait été justement de m'imposer la plus stricte obéissance à ces éclats de réalité, ne plus travailler qu'avec ces images qu'on trouve comme ça, ces bouts de phrase qu'on entend dans la rue, la stricte obéissance a la pauvreté même de tout cela. Et puis dans le livre les inscrire à travers leur manque même, leur impuissance (Bon, 1989, p. 76).

Se référant aux enquêtes menées dans les zones industrielles françaises, lieux où se développent la plupart des drames de ses œuvres littéraires. Dans cette perspective, l'écrivain vise à saisir la matérialité du monde et à devenir un témoin qui réussit dans son propos d'appréhender la réalité qui marque son époque : "le bruit de la ville tel qu'il vous parvient par la fenêtre" (Bon, 2004, p. 42). Ceci veut dire pour l'auteur de Sortie d'Usine et Décor Ciment, qu'il faut "extorquer au réel ce sentiment de présence" (p. 102) lors que le mouvement routinière de la vie quotidienne tombe dans le vide, dans le silence et surtout dans I'oubli. Cette idée configure l'écriture romanesque comme le lieu du prolongement du réel, l'espace où les drames des gens banals retrouvent un écho, voire une continuation, un nouveau moyen d'existence.

Egalement, dans l'effort pour proposer une forme esthétique renouvelée de saisie du réel, les fictions contemporaines se sont emparées massivement de faits divers susceptibles d'éclairer les manifestations les plus aigües de la société actuelle et les drames plus complexes de la vie quotidienne. Des romans comme Thérèse Desqueyroux de Mauriac ; Crime de Quinette de Romains, I'Adversaire d'Emmanuel Carrère, Viol de Daniele Sallenave, Mariage mixte de Warc Weitzmann parmi tant d'autres; ainsi que des nouvelles (La Ronde et autres faits divers de Le Clézio) et des pièces de théâtre (Les Bonnes de Genet) ont été le résultat d'une exploration littéraire dont le point de départ est le fait divers. Ces œuvres contemporaines tirent leur origine d'un évènement déclencheur qui s'enracine dans le réel et permettent de déterminer ce que celles-ci révèlent de l'état présent de la société. Néanmoins, un tel intérêt n'est pas nouveau : la littérature au XIX siècle en faisait déjà son inspiration. A cette époque-là, le fait divers était un "matériau d'incitation à la mise en récit" (Hamon, 1997, p. 10). Balzac, Stendhal, Flaubert, Maupassant, y ont volontiers recours (Viart et Vercier, 2008, p. 230), s’interrogeant ainsi sur les phénomènes de la 
société et la possibilité d'une lecture romanesque du monde qui aujourd'hui même se trouve au cœur de la production littéraire.

De ce point de vue, la littérature, mais surtout le roman comme le genre privilégié des poétiques réalistes tout au long de I'histoire, répond toujours donc à l’appel du réel - tel que cet appel s'adresse à chacun dans l'expérience de son existence dans un milieu retracé par les lettres. Le regard qui porte sur l’œuvre une poétique basée sur le réel permet donc à la fois de se figurer les faits politiques, historiques et économiques qui ont marqué une période de temps déterminée et de mener une réflexion argumentée sur la vie à travers la mise en scène de personnages qui, hier comme aujourd'hui, se sont battus contre un système qui les dépasse.

Dans ce sens par exemple, et face à l'affaire Romand $^{4}$, l'écrivain Emmanuel Carrère s'interrogent sur les forces du mal qui ont poussé un homme normal, époux et père de famille, à devenir assassin. Dans ce cas-là, le récit littéraire se consolide non seulement dans le cadre d'un souci de réalité qui se penche sur la compréhension des faits, tel qui s'est passé au XIX siècle, mais dans une démarche où l'on vise à s'emparer de ce que l'on dit à propos des évènements. C'est ainsi que le roman devient l'espace de parole où la conscience du sujet contemporain s'exprime sur le réel, ce qui explique que les écrivains, lors des enquêtes préliminaires à l'écriture du roman, cherchent à obtenir des témoignes des personnes directement concernées. Ce processus est retracé dans le texte ainsi que les pensées de l'auteur là-dessus:

L'enquête que j'aurais pu mener pour mon compte, l'instruction dont j'aurais pu essayer d'assouplir le secret n'allait mettre au jour que des faits. Le détail des malversations financières de Romand, la façon dont au fil des ans s'était mise en place sa

4 Jean-Claude Romand est connu pour avoir menti à ses proches pendant 18 ans. II a inventé une profession de médecin-chercheur à l'OMS et pour avoir assassiné sa femmes, ses enfants et ses parents en janvier 1993. Il parait qu'il étaient sur le point de découvrir la vérité lors de leur mort. double vie, le rôle qu'y avait tenu tel ou tel, tout cela, que j'apprendrais en temps utile, ne m’apprendrait pas ce que je voulais vraiment savoir : ce qui se passait dans sa tête durant ces journées qu'il était supposé passer au bureau ; qu'il ne passait pas, comme on l'a d'abord cru [...] Cette question qui me poussait à entreprendre un livre, ni les témoins, ni le juge d'instruction, ni les experts psychiatres ne pourraient y répondre, mais soit Romand lui-même, puisqu'il était en vie, soit personne (Carrère, 2000, p. 35).

L'expérience littéraire de Carrère, et celle de tant d'autres écrivains dont l'objectif d'écriture demeure plus interrogatif que descriptif par rapport à l'extraordinaire du fait divers, suppose le consentement à une vie partagée. "L'auteur, en un sens, est devenu un personnage de son propre roman, il se met lui aussi à exister "entre deux mondes ", entre le monde de la fiction et le monde vrai auquel il appartient encore un temps. C'est sur ce modèle que le lecteur va plus tard se couler. Ce battement du réel et de l'imaginaire qui nous saisit pendant la lecture est l'essence de la fiction dramatique ou épique (Sallenave, 1991, p. 132).

Ces déplacements entre deux mondes sont véhiculés par l'imagination du romancier, "son imagination loin de déformer la réalité, doit la pénétrer en profondeur et révéler cette réalité à elle-même, avec la force des infrarouges et des ultraviolents pour détecter ce qui se cache derrière les apparences ", (Modiano, 2010) et dévoiler les énigmes de notre société. Cela dit, la nature critique de la fiction contemporaine est donc attentive aux faits mais convoque également la pensée d'une époque et d'une société éclairée par la lumière révélatrice de l'écrivain contemporain : celui qui reconnait, comprend et rend visible la dimension socio-historique des faits.

\section{CONCLUSION}

L'on a pu constater à travers cet article qu'une des grandes ambitions de la littérature est de pouvoir rendre compte du monde, tant de son milieu naturel que du milieu social. Un très grand nombre 
d'œuvres littéraires de différents genres, espaces de fiction enracinés dans le réel, en sont la preuve. Ce désir de représenter le monde est sans doute un héritage de la pensée aristotélicienne, référence fondamentale étant donné que pour ce philosophe, I'art est I'imitation de la nature. Depuis la tradition théâtrale grecque, en passant par le Moyen Age, la Renaissance, le Siècle des Lumières et le XIX, XX et XXI siècle, l'écriture du réel s'est développée à travers les âges jusqu'à se consolider comme un courant privilégié de la littérature occidentale emmaillée de nuances thématiques et esthétiques. De ce fait, la poétique du réel se renouvelle jour après jour et évolue en articulation avec l'Histoire, la Politique et pourquoi pas, l'Économie.

Ainsi, on trouve que l'écriture du réel se distingue par sa révélation d'une prise de position de I'individu face à sa réalité à travers la littérature. Ce positionnement dépasse bien évidement la description de la vie quotidienne encadrée dans un milieu social particulier en exploitant des formes romanesques mais aboutit à proposer une réflexion approfondie du réel, analyse qui constitue un discours critique, explicite ou implicite, dans lequel les rapports des êtres humains avec leur époque et leur entourage sont l'objet de la création et de la contestation. L'écriture du réel est donc un des éléments dont l'homme dispose pour mettre en question les phénomènes sociaux, historiques et politiques qui le touchent et pour se battre contre une réalité qui l'anéantit. Dans ce sens, le fait de proposer un portrait du monde entraine le besoin de comprendre le regard critique de l'écrivain qui cherche, en montrant la réalité telle qu'elle est, à faire connaitre les mœurs d'une certaine époque mais aussi à susciter une réflexion vis-à-vis de ces habitudes. Il s'agit donc d'une manière de repenser le monde.

"Que ce soit le réalisme de l'observation (Balzac), le réalisme de la perception (Stendhal et Proust), le réalisme du sentir (Flaubert), le réalisme visionnaire (Hugo, Zola) (Vasseviere et Toursel, 2011, p. 151), ou le néoréalisme du XX et XXI siècle", tous ces formes d'écritures du réel répondent, à des degrés divers, à l'ambition du départ : s'emparer de la réalité, pour fabriquer des univers collectifs, dont le réfèrent est un milieu concret, où les personnages sont en quête de comprendre les contraintes d'une société qui les dépasse.

C'est pourquoi on considère que l'objet d'intérêt du roman, en tant que genre privilégié de l'écriture du réel dans toutes ses formes, est I'homme saisi dans sa difficulté d'habiter le monde. II est en effet centré sur la problématique de l'individu confronté à la société, de son rapport à l'ordre préétabli, partout présent, de son désir d'habiter un monde hostile. Cette préoccupation constitue dans les termes de Thomas Pavel (2003) la Pensée du roman.

Néanmoins, "la mobilité des écritures face au réel, la versatilité des styles, les revirements de manière, ces langages pluriels ne désignent-ils pas la radicale impossibilité de définir, de fixer le réel ?" (Dufour, 1998, p. 315) Baudelaire avait déjà posé la question autrement : I'art, la littérature transcrivant le réel sont-ils une illusion, ou une impossibilité ? Un siècle plus tard, Camus reconnait pourtant que si "en fait l'art n'est jamais réaliste, il a parfois la tentation de l'être" (Camus, 1951, p. 15). En effet, si l'on reconnait que la subjectivité de l'écrivain est une sorte de filtre, malgré les prétentions d'objectivité, et que le regard de l'auteur et du lecteur apportent des nuances à la réalité reflétée dans l'œuvre littéraire, on serait toujours d'accord sur le fait que le réel a une dimension simple, banale mais également énigmatique et mystérieusement insaisissable qui dépend de la réflexion et de la perception de celui qui écrit. Dans ce sens, la littérature ne transfigure le réel ni le représente mais permet de se le figurer, de le repenser et de l'explorer. Ceci est à notre avis, I'un des plus puissants moteurs de la littérature : l'impossibilité de saisir la réalité dans tout son ensemble fait que l'on puisse la reprendre sans fin, en revenir sans cesse. Le réel, dans son rapport avec la littérature, nous amène toujours à le revisiter, à l'explorer à nouveau, à le repenser en interrogeant le temps, les époques, 
les mœurs et les systèmes. Cet espace éphémère que l'on appelle le réel s'ouvre incessamment vers l'écriture en lui donnant chaque jour une nouvelle possibilité de s’éclater dans la quête du reflet du monde. La tension entre l'œuvre et le réel sera toujours la source des questionnements et réflexion en tant qu'élément fondamental de la création littéraire : la discussion est loin d’être achevée.

\section{REMERCIEMENTS}

Cet article est issu du projet de recherche: Figures, thèmes et valeurs, développé en 2014 à l'Universidad del Valle et inscrit dans la "vicerrectoría de investigaciones" avec le code 4315.

\section{RÉFÉRENCES BIBLIOGRAPHIQUES}

Aristote. (1990). La Poétique. Paris: LGF, le livre de poche classique.

Balzac, H. (1831). La Peau de chagrin. Paris: Editorial Garnier.

Balzac, H. (1842). La Comédie Humaine. [En ligne]. Disponible sur: http://gallica.bnf.fr/ark:/12148/bpt6k101322t.

Baudelaire, C. (1859). Salon de 1859. [En ligne]. Disponible sur: http://charles.baudelaire.perso.sfr.fr/Salon1859/Salon1859.php.

Barthes, R. (1968). L'Effet de Réel. [En ligne]. Disponible sur: http://www.persee.fr/web/home/prescript/Article/ comm_0588-8018_1968_num_11_1_1158.

Becker, C. (2005). Lire le Réalisme et le Naturalisme. Paris: Armand Colin.

Bon, F. (2004). Daewoo. Paris: Fayard Livre de Poche.

Camus, A. (1951). L'homme révolté. Paris: Gallimard.

Carrère, E. (2000). L'Adversaire. Paris: P.O.L.

Cousin, V. (1845). Du beau et de l'art. Revue des Mondes, 9, 793-830.

Champfleury, J. (1857). Le Réalisme. Paris: M. Lévy Frères.

Diderot, D. (1761). Éloge de Richardson. Disponible sur: http://www.tc.umn.edu/ dbrewer/French8270/ Diderot.pdf
Duflo, C. (2013). Roman, morale et vérité. Littérature, $171,3-12$

Dufour, P. (1998). Le Réalisme. Paris: PUF.

Hamon, P. (1997). Fait divers et littérature. Romantisme, 97, 7-15.

Hugo, V. (1827). CEuvres complètes II. Paris: Gallimard, Bibliothèque de la Pléiade.

Kaplan, L. (1982). L'Excès d'usine. Paris: P.O.L.

Maupassant, G. (1888). Pierre et Jean. Paris: Albin Michel.

Mizuno, H. (2005). Nerval face au réalisme. Les nuits d'octobre et l'esthétique nervalienne. Revue d'histoire littéraire de France, 822, 817-841.

Modiano, P. (2010). Discours de réception du Prix Nobel. [En ligne]. Disponible sur: http://www. lemonde.fr/prix-nobel/article/2014/12/07/verbatim-le-discours-de-reception-du-prix-nobel-de-patrick-modiano_4536162_1772031.html

Mordillat, G. (2004). Les Vivants et les morts. Paris: Calmann-Lévy Livre de Poche.

Pavel, T. (2003). La pensée du roman. Paris: Gallimard.

Perec, G. (1992). Pour une littérature réaliste. Paris: Seuil.

Platon. (1991). La république. Paris: G.F. Flammarion.

Proust, M. (1927). Le temps retrouvé. Paris: Gallimard, Bibliothèque de La Pléiade.

Revue L'Infinie (1989). Interview avec François Bon. N. 27, 70-78.

Robbe-Grillet, A. (1961). Pour un Nouveau Roman. Paris: Minuit.

Sallenave, D. (1991). Les Dons des morts. Sur la littérature. Paris: Gallimard.

Sartre, J. (1948). Qu'est-ce que la littérature? Paris: Folio Essais.

Stalloni, Y. (2011). Ecoles et courants littéraires. Paris: Armand Colin.

Stendhal. (1972). Le Rouge et le Noire. Paris: Classiques, Le livre de Poche.

Vassivière, J. et Toursel, N. (2011). Littérature: 40 textes théoriques et critiques. Paris: Armand Colin.

Viart, D. et Vercier, B. (2008). La littérature française au présent. Paris: Bordas.

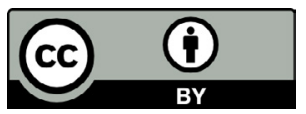

[ 211 ]

enunciación

ISSN 0122-6339 • ISSNe 2248-6798 • Vol 21, No 2 (julio-diciembre 2016). pp. 201-211. 